\title{
Yong-Sok Ri
}

Kim Il Sung University Pyongyang

the Democratic People's Republic of Korea

\section{MODUS PONENS AND MODUS TOLLENS: THEIR VALIDITY/INVALIDITY IN NATURAL LANGUAGE ARGUMENTS}

\begin{abstract}
The precedent studies on the validity of Modus ponens and Modus tollens have been carried out with most regard to a major type of conditionals in which the conditional clause is a sufficient condition for the main clause. But we sometimes, in natural language arguments, find other types of conditionals in which the conditional clause is a necessary or necessary and sufficient condition for the main clause. In this paper I reappraise, on the basis of new definitions of Modus ponens and Modus tollens, their validity/invalidity in natural language arguments in consideration of all types of conditionals.

Keywords: affirming the antecedent, affirming the consequent, argumentation, denying the antecedent, denying the consequent, modus ponens, modus tollens, validity.
\end{abstract}

\section{Introduction}

Some logicians may be surprised at the word "invalidity" in the title of this paper because the leading logic textbooks say that Modus ponens and Modus tollens are deductively valid. Consider the following passages from [Layman, 2002].

\section{Modus Ponens}

1. If it is raining, then the ground is wet.

2. It is raining.

So, 3. The ground is wet.

This argument is obviously valid: On the assumption that its premises are true, its conclusion must be true also. Using letters to stand for statements, the form of the argument is as follows: 
Yong-Sok Ri

Modus Ponens

1. If $\mathrm{A}$, then $\mathrm{B}$.

2. A

So, 3. B.

(A stands for "it is raining"; B stands for "the ground is wet.") This form of argument is always valid.

Modus Tollens

1. If $\mathrm{A}$, then $\mathrm{B}$.

2. Not B.

So, 3. Not A.

Every argument having the form modus tollens is valid. [Layman, 2002, pp. 22-24]

This is not Layman's personal opinion but a conventional dogma in the sphere of formal logic. It has been developed with main focus on if-then statements in natural languages. I fully understand the reason for focusing on if-then statements. The basic reason, I think, is that if-then statements are the vast majority of conditional statements used in the speech act. How frequently do we utter conditional statements? What percentage of the conditional statements used in the speech act are if-then statements? Getting interested in these questions, I myself searched for instances of conditional statements and if-then statements in 1310 documents stored on my laptop. The documents were all papers on logic, rhetoric, or argumentation theory. The following statistical information can presumably provide some cues, albeit not representative enough to draw any general conclusions regarding conditionals. The search result showed 116771 instances of conditionals and 115782 if-then statements found. In average, about 90 instances were found in each document. And the result shows that about 99 percent of the conditional statements were if-then statements. I acknowledge that the samples for my statistics are not representative and that the frequency of conditional statements and percentage of if-then statements depend upon various factors such as scope, personal speech habit, etc. Anyhow, as far as I searched, if-then statements are the vast majority of the conditional statements used in the speech act. This is why it seems very likely that most logicians focus on if-then statements.

On the other hand, my computerized search also showed a minority of the conditional statements having an only if-clause or an if and only if-clause as their components. 555 instances of an only if-clause were found in 332 documents and 434 instances of an if and only if-clause in 132 documents. The percentage of only if-clauses and if and only if-clauses was incompara- 
bly smaller than that of if-clauses. But here a question is raised. Why did the authors use conditional statements having an only if-clause or an if and only if-clause? Surely because the conjunctions "if", "only if" and "if and only if" have different syntactic functions. If-then statements indicate implication whereas only if-clauses indicate prerequisite. And the conditional statements having an if and only if-clause indicate both implication and prerequisite. In other words, the logical relationships between conditional clause and main clause vary from conditional to conditional. That is why the writers used an only if-clause or an if and only if-clause in order to express prerequisite or both.

It is, I think, crucial to differentiate implication from prerequisite in translating any conditionals into a foreign language. As far as I have seen, some students would confuse prerequisite with implication and make some mistakes in Korean-English translation. Moreover, the conjunction "only if" is less familiar than "if" to English learners. So some students said "if there is water, then fish farming is possible." This English sentence is wrong because water is a prerequisite for fish farming. Thus they should have said "Only if there is water, is fish farming possible." This example suggests that we should differentiate the conditional statements having an only if-clause or an if and only if-clause from if-then statements, even though they are not often used.

In this paper, I consider three types of conditional statement in which the antecedent is a necessary, sufficient, or necessary and sufficient condition for the consequent. This classification seems incompatible with the prevailing position that modus ponens and modus tollens are always valid. Especially when a conditional statement has an only if-clause, things are different. For the purpose of resolving the incompatibility, first I examine and bring back the original meanings of modus ponens and modus tollens. Their conventional definitions used in formal logic are replaced by wider definitions. Second, I carry out a new evaluation of their validity/invalidity in natural language arguments.

\section{The precedent positions on modus ponens and modus tollens}

There has been lots of discourse on modus ponens and modus tollens among scholars who research logic, dialectics, rhetoric, or argumentation theory. In summary, there are four main positions.

First, modus ponens is identified with affirming the antecedent (AA from now on) and modus tollens is identified with denying the consequent 
(DC from now on), which is suggested by two parentheses in the following passage extracted from the Stanford Encyclopedia of Philosophy 2011.

Especially when one considers non-fallacy approaches to informal argument, one might compare informal logic to classical formal logic. In both cases one finds an attempt to identify general criteria for good reasoning and argument schemes that incorporate specific forms of reasoning. In the latter case, this is reflected in a focus on validity and soundness, and on deductive argument schemes encapsulated in rules of inference like modus ponens ("Affirming the Antecedent"), double negation, modus tollens ("Denying the Consequent"), etc. [Groarke, 2011, pp. 17-18]

According to some other encyclopaedias, the leading logic textbooks, and references, the term modus ponens is a synonym of AA and modus tollens is a synonym of DC. The passages above from [Layman, 2002] are good examples, too. And the following two sentences are in the explanation of the entries Modus Ponens and Modus Tollens in the New World Encyclopedia 2008.

Modus Ponens is referred to also as Affirming the Antecedent and Law of Detachment. MT is often referred to also as Denying the Consequent.

Second, modus ponens and modus tollens are universally regarded as valid forms of argument.

A valid argument is one in which the premises support the conclusion completely. More formally, a valid argument has this essential feature: It is necessary that if the premises are true, then the conclusion is true. [Layman, 2002, p. 3] According to this definition of valid argument, modus ponens and modus tollens guarantee a true conclusion, provided the premises are true. This position is based on the following theory.

Explanations of the standard, deductivist classification of conditional arguments begin with the claim that conditional assertions occurring in natural language arguments are to be interpreted as asserting a materially [or factually] sufficient/necessary relationship between the components of the conditional. Conditional assertions can be standardized into a natural language expression of the form "If A then C" where $\mathrm{A}$ and $\mathrm{C}$ are variables for natural language statements. A is the antecedent of the conditional, and marks a sufficient condition for C [the consequent of the conditional]. Similarly, the consequent, C, marks a necessary condition for the antecedent A. As such, expressions of the form "If A then C" assert a relationship between the components of the conditional. This relationship is that $\mathrm{A}$ is sufficient for $\mathrm{C}$ and that $\mathrm{C}$ is necessary for A. [Godden \& Walton, 2004, p. 220] 
On the ground that $\mathrm{A}$ is a sufficient condition for $\mathrm{C}, \mathrm{AA}$ is always regarded as a valid form of argument.

Similarly, provided that $\mathrm{C}$ is a necessary condition for A, the conclusion denying the antecedent necessarily follows the premise denying the consequent. So denying the consequent is always regarded as another valid form of argument. This view is shown by the following passage from New World Encyclopedia 2008.

Modus Tollens (Latin for "mode that denies" abbreviated as MT) is another form of valid inference. As in the case of MP, an instance of MT inferences involves two premises. One is again a conditional statement If $A$ then $B$, while the other, unlike MP, is the negation of the consequent, i.e. a statement of the form not $B$. From such pairs of premises, MT allows us to infer the negation of the antecedent of the conditional statement, i.e. not $A$. To see the validity of such inferences, assume toward contradiction that $A$ is true given the two premises, If $A$ then $B$ and not $B$ are true. Then, by applying MP to $A$ and If $A$ then $B$, we can derive $B$. This is contradictory and thus $A$ is false, i.e. not $A$.

Conviction for the validity of modus ponens and modus tollens can be found in [Burke, 1994]. In "Denying the Antecedent: A Common Fallacy?" he puts non-fallacious interpretation on 5 argumentative passages that appear to be instances of denying the antecedent.

Each of our passages (except 6) contains an argument. But in no case is there adequate reason to consider the conditional a part of the argument. In each case it is at least as plausible to ascribe to the conditional some other role. In each case it is at least as plausible to take the argument to be an enthymematic instance of modus ponens (or of modus tollens, depending on the formulation of the unstated conditional). [Burke, 1994, p. 2]

His non-fallacious interpretation seems to be based on his strong belief in the validity of modus ponens and modus tollens. In other words, he argues that the passages are not fallacious on the ground that they are instances of modus ponens or modus tollens.

As seen above, modus ponens and modus tollens are usually regarded as valid forms of argument on the ground that the antecedent is a sufficient condition for the consequent.

As a matter of fact I know an exception in [Walton, 2002]. We can find a position different from the common view on modus ponens in "Are Some Modus Ponens Arguments Deductively Invalid?"

What is argued below, however, is that there are many common arguments used daily in everyday reasoning that have the form modus ponens but are not deductively valid. [Walton, 2002, p. 19] 
Walton's view on the invalidity of modus ponens is grounded on new classification of conditional statements. He divides conditionals into three types: the absolute, probabilistic, and abductive (defeasible or plausibilistic) conditional. [Walton, 2002, p. 30] In cases that the first premise is the probabilistic or abductive conditional, he suggests, modus ponens is deductively invalid. But he also admits that modus ponens is valid in the case of an absolute conditional.

The new view will restrict the applicability of deductive logic to modus ponens arguments in which the conditional is of an absolutistic sort only. [Walton, 2002, p. 44]

Third, denying the antecedent (DA) and affirming the consequent (AC) are regarded as invalid forms. (It may come as a surprise that I talk about $\mathrm{DA}$ and $\mathrm{AC}$ in this article on modus ponens and modus tollens. Section 3 will help you to understand the reason.)

A formal fallacy is understood as an argument which is invalid according to some logical system. Amongst fallacies which do not follow the rules of classical propositional logic and are claimed to be common in natural dialogues are, e.g., fallacies of incorrect operations on implication, i.e. denying the antecedent $(\varphi \rightarrow \psi, \neg \varphi$, therefore $\neg \psi)$ and affirming the consequent $(\varphi \rightarrow \psi, \psi$, therefore $\varphi$ ). [Yaskorska, et al., 2012]

DA is universally recognized as a formal fallacy in reasoning because arguments using this form of reasoning are invalid. It is possible for them to have true premises but a false conclusion. [Stone, 2012] In arguments having the form of DA the minor premise suggests that a sufficient condition for the consequent is not provided. But other sufficient conditions might be provided. Therefore, negation of the consequent cannot be established on the ground that a sufficient condition for the consequent is not provided. Such a view is also expressed in the following passages from [Orsinger, 2011]

7. The Fallacy of Denying the Antecedent.

There are two Fallacies of Implication. The first is the Fallacy of Denying the Antecedent which occurs when disproving the Antecedent of a Conditional Proposition (if $\mathrm{P}$ then Q) is taken as proof that the Consequent is false. Disproving the Antecedent does not prove that the Consequent is false. It only establishes that the Implication does not apply to this particular situation. Fallacious example:

$\mathrm{P}$ implies Q.

$\mathrm{P}$ is false.

Therefore, Q is false. [Orsinger, 2011, p. 46] 
As for AC, when the consequent is a necessary condition for the antecedent in the conditional premise (the major premise), the minor premise suggests that one of the necessary conditions for the antecedent is provided. But only one necessary condition cannot cause a result. Only when all of the necessary conditions are provided, can a result be brought out. So it is possible that the premises are true and the conclusion is false. In some cases, $\mathrm{AC}$ is regarded as a fallacy. The following quotations are good examples.

\section{The Fallacy of Affirming the Consequent}

The second Fallacy of Implication is Affirming the Consequent. This logical fallacy, identified by Aristotle, occurs when someone concludes that, because "P implies Q", therefore "Q implies P". The term "Affirming the Consequent" comes from the fact that "the Consequent" in the conditional clause, which is "Q", has been "affirmed," or proven to be true. This Fallacy is also known as Converse Error. The Fallacy is expressed: "If A then B. B is true. Therefore, A is true." Fallacious example:

(1) If P, then Q. (1) P implies Q.

(2) Q. or

(2) Q.

(3) Therefore, P.

(3) Therefore, P.

We can put the discussion into the context of cause and effect. Where there are several possible causes of a particular effect, the existence of that effect cannot itself establish which cause is involved. [Orsinger, 2011, p. 46]

On the other hand, we can also find slightly different views about DA in [Burke, 1994] and [Moldovan, 2009].

Burke raises a problem whether DA is a common fallacy or not and puts an alternative interpretation on some argumentative passages that appear to be instances of denying the antecedent. In his opinion, the conditional contained by the passage is a preface to the argument rather than a premise of it. On the basis of this interpretation, he argues that the passages cannot fairly be charged with the fallacy of denying the antecedent. Finally, he says that he was unable to find a single published argument that can justifiably be charged with denying the antecedent. [Burke, 1994]

Moldovan is concerned with the analysis of fragments of a discourse or text that express arguments suspected of being denials of the antecedent. He focuses on pragmatic aspects of argument analysis with respect to the identification of the premises of an argument. Appealing to a Gricean account of the pragmatics of conditionals, he shows that some such fragments express arguments that are valid, and do not instantiate DA. [Moldovan, 2009] In conclusion, both Burke and Moldovan give non-fallacious interpretation of some instances which can be said to have the form of DA from the perspectives of formal logic. 
Anyhow, they regard the instances as being valid on the ground that they do not really have the forms of DA. This shows that they regard DA itself as invalid forms.

As seen above, AC and DA are recognized as invalid forms on the basis of the theory that the antecedent is a sufficient condition for the consequent.

Fourth, some scholars suggest that invalid forms can be effectively used in argumentation. This position comes rather from informal logic and pragma-dialectics than from formal logic.

Some scholars regard DA as a legitimate and effective strategy for undermining a position. In "Denying the Antecedent as a Legitimate Argumentative Strategy: A Dialectical Model", Godden and Walton argue that DA is not always a fallacious argumentative strategy. Instead, they suggest, there is a legitimate usage of DA according to which it is a defeasible argument against the acceptability of a claim. The dialectical effect of denying the antecedent is to shift the burden of proof back to the original proponent of a claim. They provide a model of this non-fallacious usage which is built upon pragmatic models of argumentation. [Godden and Walton, 2004, p. 219] Stone also recognizes the legitimate usage of DC. He argues that denying the antecedent provides inductive support for rejecting a claim as improbable." [Stone, 2012, p. 327] In "Logical Fallacies as Informational Shortcuts" Floridi uses a Bayesian analysis to argue that denying the antecedent and affirming the consequent "are not just basic and simple errors, which prove human irrationality, but rather informational shortcuts, which may provide a quick and dirty way of extracting useful information from the environment" [Floridi, 2009, p. 317]. In addition, Walton makes the assertion that some invalid subtypes of modus ponens perform a useful function in arguments from sign. He takes the example of the Measles Inference.

If a patient has red spots (of a certain kind), then the patient has measles. This patient has red spots (of this certain kind).

Therefore, this patient has measles.

It is a typical kind of inference very commonly used in medical diagnostics [Fox and Das, 2000]. It can also be classified as an instance of argument from sign. ... The function of the inference is to make a guess or hypothesis that can lead to testing. Once the tests are in, the findings may confirm the guess, or they may show it was false. Either way, knowledge is gained about the patient's diagnosis. If the initial guess can be ruled out, then other diagnoses can be explored and tested. If the guess turned out to be right, then treatment for measles can be undertaken, and the possibility of having to deal with other possible diseases can be set aside. So even though the inference is not deductively valid, it performs a very useful function as a kind of reasoning in medical diagnosis. [Walton, 2002, p. 32] 
"Modus ponens" and "Modus tollens": Their Validity/Invalidity in Natural...

To summarize, AA and DC are regarded as valid forms, whereas DA and $\mathrm{AC}$ are invalid but sometimes effectively used in argumentation and research. I definitely agree with them in case that the antecedent is a sufficient condition for the consequent. However, the antecedent can be a necessary condition or a necessary and sufficient condition for the consequent. Things are different in those cases, which is detailed in Section 4.

\section{New definitions of modus ponens and modus tollens}

In natural language arguments we sometimes find or use conditional statements having an only if-clause as the antecedent.

The Combustion Example:

Only if there is oxygen, can combustion occur.

The match lit (combustion occurred) in the second bottle.

Thus there was oxygen in it.

In this example, the first premise is a conditional statement. It consists of two clauses: an only if-clause and a main clause. Which clause do you think is the antecedent? The former is certainly the antecedent and the latter the consequent. The second premise is affirming the consequent and the conclusion is affirming the antecedent. So this argument can be said to have the form of AC. Here a problem is raised. AC has been recognized as an invalid form. But in this argument it is impossible that the premises should all be true while the conclusion is false. In order to solve this problem I suggest classifying $\mathrm{AC}$ as a subtype of MP. That is, MP includes AC as well as AA. I find this definition consistent with the original meaning of MP described in some encyclopaedias, textbooks, and papers.

Modus Ponens (Latin: mode that affirms; often abbreviated as MP) is a form of valid inference. [New World Encyclopedia 2008]

Affirming the Antecedent (Modus Ponens).

Modus ponendo ponens (in English, "the way that affirms by affirming") is a particular form of Conditional Proposition. [Orsinger, 2011, p. 23]

We need to pay attention to the word origin in the parentheses. As you can see, the name "modus ponens" stems from the Latin words "modus ponendo ponens" which means "the way that affirms by affirming". Then, which can we affirm, antecedent or consequent? Both are possible, that is, 


\section{Yong-Sok Ri}

we have two ways: affirming the antecedent (AA) and affirming the consequent (AC). But in spite of these two possible ways, some logic textbooks and references say that MP equals AA. Why do they identify MP with AA? I think there are two reasons. One reason is that they usually consider just conditional statements which have an if-clause as the antecedent. In such conditional statements the if-clause usually expresses a sufficient condition for the result expressed by the main clause. Consider the following conditional statement treated in [Layman, 2002]

If it is raining, then the ground is wet.

According to him, statements (a) through (f) following are all stylistic variants of the above conditional statement, that is, alternate ways of saying the same thing:

a. Given that it is raining, the ground is wet.

b. Assuming that it is raining, the ground is wet.

c. The ground is wet if it is raining.

d. The ground is wet given that it is raining.

e. The ground is wet assuming that it is raining.

f. It is raining only if the ground is wet. [Layman, 2002, p. 21]

He says that each of the above statements is logically equivalent to "If it is raining, then the ground is wet." "If" and its stylistic variants in statements (e.g., "given that" and "assuming that") introduce an antecedent. But "only if" performs a function different from the other variants. Layman clarifies its logical force as follows:

When combined with "only," as in (f), the situation alters dramatically. Statement (f) has the same logical force as (45), but the phrase "only if" is confusing to many people and bears close examination.

To clarify the logical force of "only if," it is helpful to consider very simple conditionals, such as the following:

46. Rex is a dog only if Rex is an animal.

47. Rex is an animal only if Rex is a dog.

Obviously, (46) and (47) say different things. Statement (47) is false. Rex may well be an animal even if Rex isn't a dog. Thus, (47) says, in effect, that "If Rex is an animal, Rex is a dog." But (46) says something entirely different, and something true - namely, that if Rex is a dog, then Rex is an animal. In general, statements of the form "A only if B" are logically equivalent to statements of the form "If A, then B." They are not logically equivalent to statements of the form "If B, then A." Another way to generalize the point is to say that "only if" (unlike "if") introduces a consequent, that is, a then-clause. [Layman, 2002, p. 21-22] 
Applying his interpretation to "Only if there is oxygen, can combustion occur," you can regard the main clause as the antecedent and the only if-clause as the consequent. In this case the form of reasoning is converted to AA. Thus AA and AC can be symbolized by means of the same formula. Surely, this idea is originated from the perspective of formal logic. This perspective is another reason for identifying MP with AA.

By the way, a question is raised from his interpretation. Why does he interpret the only if-clause as the consequent, whereas the if-clause is the antecedent? Both clauses express the condition for a certain result. An ifclause usually expresses a sufficient condition and an only if-clause a necessary condition. In the above example, oxygen is a necessary condition for combustion. And the main clause expresses an actual or possible result. In this sense, it seems more reasonable to regard the only if-clause not as the consequent but as the antecedent. Then even if the result is described by the former clause, the argument in which the second premise affirms the result has the form of AC. Therefore AC is also included in MP, a way that affirms by affirming. As mentioned above, I assert that MP includes not only AA but also AC in natural language arguments.

Similarly, I think MT includes not only DC but also DA. We need to consider the original meaning of MT. Here's a passage from [Orsinger, 2011]

Denying the Consequent (Modus Tollens).

Modus tollendo tollens (in English, "the way that denies by denying") is another form of Conditional Proposition. [Orsinger, 2011, p. 23]

As the word origin in the parentheses shows, modus tollens is literally the way that denies by denying. Then, which can we deny, the antecedent or the consequent? Both are possible. We are fully aware of the possibility of denying the consequent. The following example shows that denying the antecedent is also possible in case that the antecedent is a necessary condition for the consequent.

Only If there is oxygen, can combustion occur.

There is no oxygen on the moon.

Thus you can't light a match there.

In this sense, I contend that MT includes not only DC but also DA.

The precedent definitions of modus ponens and modus tollens seem to be narrow from the perspective of informal logic. Informal logic is an attempt to develop a logic that can assess and analyze the arguments that occur in natural language ("everyday," "ordinary language") discourse. [Groarke, 2011] 
What is the most important is that we do not always convert the conditional statement having an only if-clause to the conditional statement having an if-clause in natural language arguments. The conversion is necessary for formulation in formal logic. Without the conversion, an only if-clause can never be the consequent. To sum up, it can be said that narrow definitions of modus ponens and modus tollens come from the perspective of formal logic. That is why I suggest keeping the original meaning of the terms "modus ponens" and "modus tollens" and widening their extension in order to carry out a correct analysis of the validity/invalidity of conditional arguments in natural languages.

\section{The Validity/invalidity of modus ponens and modus tollens in cases that the antecedent is a necessary or necessary and sufficient condition for the consequent}

In natural language arguments each antecedent and consequent expresses the diversity of the contents. And it depends upon the content whether the form of a conditional argument is valid or invalid. AA and DC are valid in the cases that the antecedent is a sufficient or necessary and sufficient condition for the consequent. But they are invalid in the cases that the antecedent is a necessary condition for the consequent. Let's have a look at the following Fish Farming example.

Only if there is water, is fish farming possible.

There is enough water in my native village.

Therefore, fish farming is possible there.

You can easily understand the invalidity of this example. Even if there is water, fish farming may be impossible due to lack of any other necessary condition.

Only if there is water, is fish farming possible.

Fish farming is impossible in his native village.

Therefore, there may not be enough water.

You cannot prove lack of water on the ground of the impossibility of fish farming. Impossibility may come from absence of any other necessary conditions. These examples show that AA and DC are invalid in the cases that the antecedent is a necessary condition for the consequent. Thus in these cases you cannot establish the conclusion on the ground of the given premises. 
On the contrary, AC and DA are valid in the same cases. This is the most important point in this article.

\author{
Affirming the consequent \\ Only if there is water, is fish farming possible. \\ My friend's native village is famous for fish farming. \\ Therefore, there must be water. \\ Denying the antecedent \\ Only if there is water, is fish farming possible. \\ There is almost no water in deserts. \\ Therefore, fish farming is impossible there.
}

In these examples water is a necessary condition for fish farming and the conclusions are necessarily derived from the premises.

Finally, I get on to the cases of necessary and sufficient condition. The following geometric arguments are typical instances of arguments which have a bi-conditional as the major premise.

Affirming the consequent

If and only if two straight lines run parallel with each other, their corresponding angles are equal. Angle $a$ and $b$ are equal. Therefore $l$ and $m$ run parallel with each other.

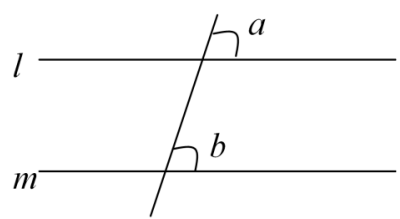

Denying the antecedent

If and only if two straight lines run parallel with each other, their corresponding angles are equal. Straight lines $l$ and $m$ don't run parallel with each other. Therefore Angle $a$ and $b$ are not equal.

These two examples show that AC and DA are valid in the cases that the antecedent is a necessary and sufficient condition for the consequent.

The following table summarizes the validity/invalidity of MP and MT.

\begin{tabular}{|l|c|c|c|c|}
\hline \multirow{2}{*}{ condition } & \multicolumn{2}{c|}{ MP } & \multicolumn{2}{c|}{ MT } \\
\cline { 2 - 5 } & AA & AC & DA & DC \\
\hline sufficient & valid & invalid & invalid & valid \\
necessary & invalid & valid & valid & invalid \\
necessary \& sufficient & valid & valid & valid & valid \\
\hline
\end{tabular}




\section{Conclusion}

The precedent views on the validity/invalidity of conditional arguments are grounded on diverse interpretations of conditional statements that are components of the arguments. Some attach most weigh to the pragmatic implicature of conditional statements while some others make great account of the dialectic role of conditionals. What is common in their diversified interpretations is to deal with and focus on if-then statements. Contrary to them, my paper is focused on only if-clauses and if and only if-clauses. Herein lies the fundamental difference between my paper and them. In natural language discourse the speakers or writers make a lot of use of conditional statements having an only if-clause or an if and only if-clause as well as an if-clause. In Asian languages such as Korean we can find conditional statements in which the former clause indicates a prerequisite more often than in English arguments. With respect to them, I widen the definitions of modus ponens and modus tollens, that is, MP includes not only AA but also AC, and MT includes not only DC but also DA.

On the basis of new definitions, I revaluate their validity/invalidity in argumentation in the cases that the antecedent is a necessary or necessary and sufficient condition for the consequent. In cases of a necessary conditional $\mathrm{AA}$ and DC are invalid, and cannot prove the conclusion. On the contrary, $\mathrm{AC}$ and DA can be used to establish a conclusion with certainty because they are valid. Due to the invalidity of modus ponens and modus tollens in some cases (including the cases of probabilistic or abductive conditional, too), I regard them merely as argumentation schemes but not as rules in natural language arguments.

\section{R E F E R E N C E S}

[Burke, 1994] Burke, M. B. (1994). Denying the antecedent: A common fallacy? Informal Logic, 16, pp. 23-30.

[Floridi, 2009] Floridi, L. (2009). Logical fallacies as informational shortcuts. Synthese, 167(2), pp. 317-325.

[Fox and Das, 2000] Fox, J. and Das, S. (2000). Safe and Sound: Artificial Intelligence in Hazardous Applications. Menlo Park, CA: MIT Press.

[Godden and Walton, 2004] Godden, D. M. and Walton, D. (2004). Denying the Antecedent as a Legitimate Argumentative Strategy: A Dialectical Model. Informal Logic, 24(3), pp. 219-243.

[Groarke, 2011] Groarke, L. (2011). Informal Logic. Stanford Encyclopedia of Philosophy http://www. plato.stanford.edu/entries/logic-informal/ 
"Modus ponens" and "Modus tollens": Their Validity/Invalidity in Natural...

[Layman, 2002] Layman, C. S. (1999). The Power of Logic. The McGraw-Hill Companies, Boston.

[Moldovan, 2009] Moldovan, A. (2009). Pragmatic Considerations in the Interpretation of Denying the Antecedent. Informal Logic, 29(3), pp. 309-326.

[Orsinger, 2011] Orsinger, R. R. (2011). The Role of Reasoning in Constructing a Persuasive Argument. http://www.orsinger.com/PDFFiles/constructinga-persuasive-argument.pdf

[Stone, 2012] Stone, M. (2012). Denying the Antecedent: Its Effective Use in Argumentation. Informal Logic, 32(3), pp. 327-356

[Walton, 2002] Walton, D. N. (2002). Are Some Modus Ponens Arguments Deductively Invalid? Informal Logic, 22(1), pp. 219-243

[Yaskorska, et al., 2012] Yaskorska, O., Kacprzak M., \& Budzynska K. (2012) Rules for Formal and Natural Dialogues in Agent Communication. In Proceedings of the international workshop on concurrency, specification and programming, pp. 416-427. Berlin: Humboldt-Universitat zu Berlin 